\title{
The Use of Video Conferencing for Persons with Chronic Conditions: A Systematic Review
}

\author{
Jennifer A. Mallow ${ }^{1 *}$, Trisha Petitte ${ }^{1}$, Georgia Narsavage ${ }^{1}$, Emily Barnes ${ }^{1}$, Elliott Theeke ${ }^{1}$, \\ Brian K. Mallow ${ }^{2}$, Laurie A. Theeke ${ }^{1}$ \\ ${ }^{1}$ School of Nursing, West Virginia University, Morgantown, WV, USA \\ ${ }^{2}$ Sovern Run, LLC, Albright, WV, USA

 \\ ebarnes@hsc.wvu.edu, etheeke@hsc.wvu.edu,bkmallow@gmail.com, Itheeke@hsc.wvu.edu
}

Received 26 April 2016; accepted 4 June 2016; published 7 June 2016

Copyright (C) 2016 by authors and Scientific Research Publishing Inc.

This work is licensed under the Creative Commons Attribution International License (CC BY). http://creativecommons.org/licenses/by/4.0/

(c) (†) Open Access

\begin{abstract}
The purpose of this paper is to present a systematic review of studies that used Video Conferencing (VC) intervention for common chronic conditions. Chronic conditions account for the majority of poor health, disability, and death, and for a major portion of health-care expenditures in the United States. Innovative methods and interventions are needed to enhance care and management, improve access to care, improve patient outcomes, narrow health disparities and reduce healthcare costs. Video Conferencing could be particularly relevant in improving health, care management, access and cost in the care of chronic illnesses. A comprehensive literature search process guided by the PRISMA statement led to the inclusion of 27 articles measuring video conferencing, at least one chronic illness, and patient outcomes for adults living in a community setting. While $\mathrm{VC}$ has been found to be feasible and effective, a low number of randomized controlled trials limit evidence. In addition, studies in this review were not designed to address the question of whether access to care in rural areas is improved through VC. Hence, more research is needed.
\end{abstract}

\section{Keywords}

Video Conferencing, Chronic Conditions, Outcomes of Care

\section{Introduction}

Living with multiple chronic conditions (MCC) compounded by experiencing health disparity is extremely "Corresponding author. 
challenging. A chronic condition is one that persists a year or longer and requires ongoing medication, attention, and/or limits activities of daily living [1]. Health disparity is an individual's ability to achieve good health outcomes, which can be affected by ethnicity, gender, age, disability, socioeconomic status, and geographic location, particularly for those living in rural areas [2] [3]. Chronic conditions account for the majority of poor health, disability, and death, and for a major portion of health-care expenditures in the United States (US) [4]. Approximately half (117 million) of US adults have at least one chronic condition, and 1 in 4 adults have multiple chronic conditions [5]. Individuals with MCC may have difficulty achieving goals of treatment due to the complexity of treatments, conflicting advice for each condition, and co-existing health disparities [6]-[9]. Therefore, persons with MCC and health disparities living in rural areas are at higher risk for limited access to care which can result in poor chronic condition control and decreased self-management [10] [11].

Hence, treating MCC while also addressing health disparity is a complicated issue for healthcare providers. This complexity has been defined as the gap between the individual's needs and the capacity of healthcare services to support those needs [6]. Innovative methods and interventions are essential to enhance the care and management of MCC, improve access to care, improve patient outcomes, narrow health disparities and reduce healthcare costs [12]. Use of technology to treat MCC has been suggested [7] because technology can improve the capability to monitor patients, coordinate care services for patients seeking multiple healthcare providers in different geographic locations, and manage patient symptoms [13]. Video Conferencing (VC) offers a potential solution to this complexity by incorporating audio/visual enhancements to technology-based interventions for the treatment of individuals with health disparity and MCC.

Video conferencing is a communication technology that allows two or more locations to connect in real-time two-way video and audio transmission [14]. This type of technology enables patients to access healthcare services, by using the internet, without the burden of distant travel. Eliminating health disparities such as advanced age, physical disability, and isolated geographic location are possible through this technology. Video Conferencing could be particularly relevant in the context of MCC care when there are high levels of unmet need, and demands on services are likely to increase [5]. The purpose of this paper is to present a systematic review of studies that used Video Conferencing intervention for common chronic conditions.

\section{Methods}

The PRISMA method [15] guided the development of this systematic review. A comprehensive literature search was conducted using EBSCOHost with the following databases selected: Academic Search Complete, CINAHL with Full Text, ERIC, MEDLINE, PsycARTICLES, and PsycINFO. The search term "Video Conferencing” was used and limits were set to include only peer-reviewed studies of adults written in the English Language. The literature search began on 5/1/2015 and concluded on 7/16/2015. The search netted 460 articles; 162 duplicates were eliminated and 298 abstracts were reviewed with the following inclusion criteria: 1) use of Video Conferencing discussed in the abstract, 2) included patients, 3) adults, 4) at least one chronic illness discussed in the abstract, and 5) outpatient/community setting. Articles were excluded if the abstract did not meet these criteria. Three authors completed the literature search (JM, BKM, \& ET) to ensure accuracy of the search. Forty abstracts met the inclusion criteria and these full text articles were obtained. The Rosswurm literature critique worksheet [16] was used to review each article in order to reduce the risk of bias of individual studies at the study level and also across all identified studies. The full text article and the literature critique worksheet were reviewed for accuracy by all authors. The results of each critique were then placed into a literature matrix, which was again reviewed by each author for accuracy. The matrix includes the authors, year of publication, location of the study, aim of the study, study design, type of video conferencing, sample size, data collection methods, chronic disease focus, and results of the study.

After reading and critiquing full articles, 13 articles did not meet the inclusion criteria and were eliminated from this review. One article did not fit the definition of VC used in this article [17]. Two articles explained the potential of VC but did not report patient outcomes [18] [19]. Four articles were study protocols and did not report data [20]-[23], three articles were commentaries or letter's to the editor [24] [25], and four articles measured provider but not patient outcomes [26]-[29]. Finally, the Cochrane Library was searched for a review on video conferencing, but no applicable results were found.

\section{Results}

A total of 27 articles are included in this review (see Figure 1). The majority of studies took place in The United 


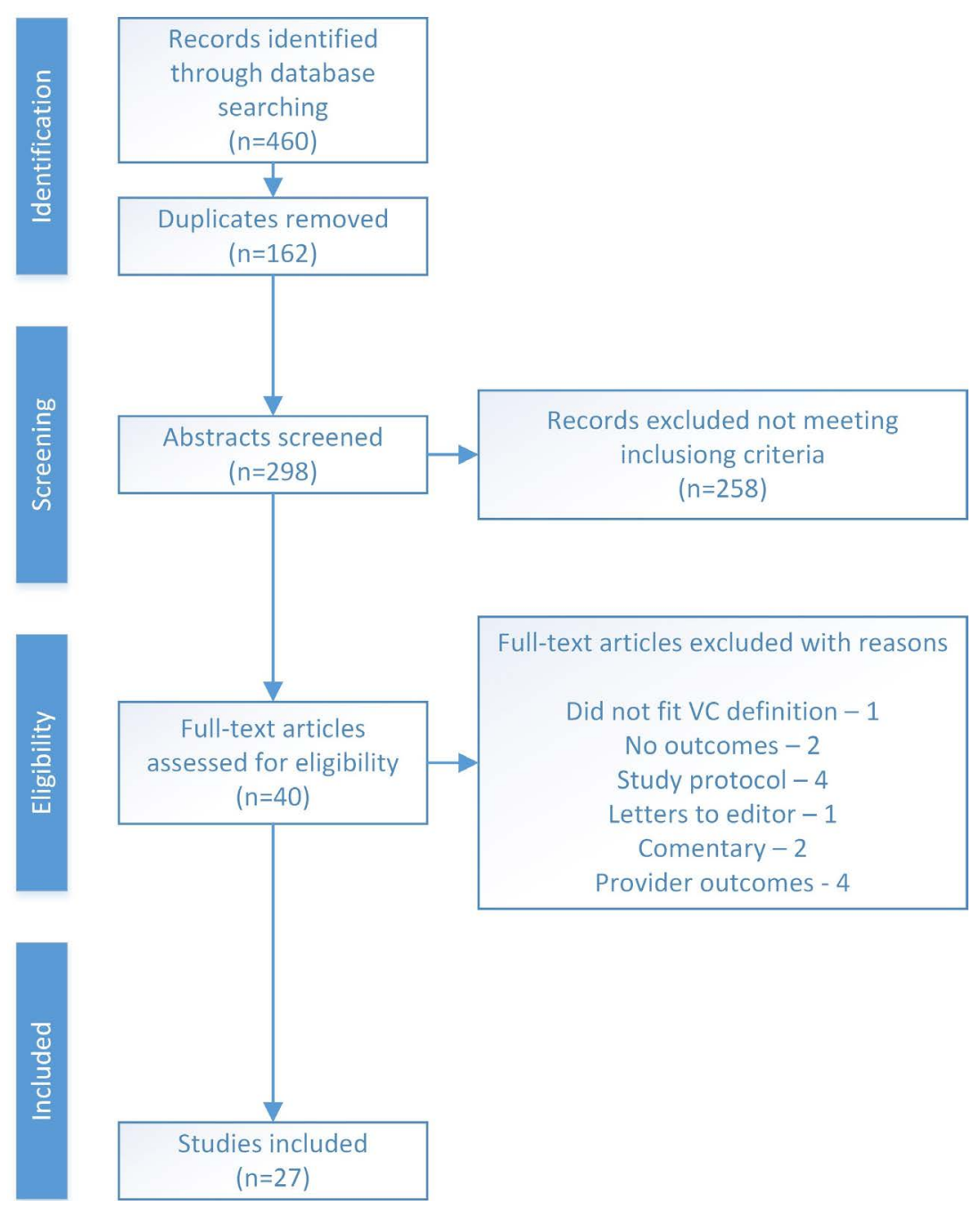

Figure 1. Literature search [insert here from uploaded. jpg file].

States $(n=11)$ and Canada $(n=9)$. While, a few articles were set in Australia $(n=3)$, The United Kingdom $(n=$ $3)$, and China $(\mathrm{n}=1)$. Limits were not set for year published while searching the literature. However, all studies in this review were published within the last 15 years and the majority were published in the last 5 years $(n=16)$. Of the reviewed articles, three were review articles and eight were randomized controlled trials. The remaining articles were feasibility or quasi-experimental studies $(n=10)$, descriptive observational studies $(n=3)$, one cost analysis, one purely qualitative article and one retrospective chart review. The sample size for articles ranged from 2 - 249 participants with the majority of studies having 50 or less participants $(n=14)$. The type of connection used for the video conferencing was often web based $(n=14)$ especially in the last 5 years (see Table 1).

\subsection{Results Overview}

Videoconferencing has been used in many different areas of healthcare and has been generally successful in improving patient outcomes. The types of chronic illness that researchers evaluated using VC included: mental health issues ( $(n=11)$, neurological $(n=6)$, long term care patients $(n=2)$, oncology $(n=2)$, rehabilitation $(n=$ $2)$, obesity $(n=1)$, angina/heart failure $(n=1)$, diabetes $(n=1)$, Human Immunodeficiency Virus $(n=1)$. Video Conferencing was shown to be feasible, result in positive patient experiences, improve outcomes, decrease hospital days, be similar to in-person care, decrease cost, and improve caregiver quality of life [23] [25] [30]-[52]. Two studies found VC to be less efficient or potentially not feasible in certain populations $(n=2)$ [53] [54]. 
Table 1. Literature review matrix: study aim, design, type of video conferencing (vc), sample, data collection characteristics, chronic illness, and results.

\begin{tabular}{cccccc}
\hline $\begin{array}{c}\text { Authors, } \\
\text { Year) } \\
\text { Location }\end{array}$ & Aim & Design & $\begin{array}{c}\text { Type of Video } \\
\text { Conferencing } \\
\text { (VC) }\end{array}$ & $\begin{array}{c}\text { Sample Size/ } \\
\text { (Description) }\end{array}$ & $\begin{array}{c}\text { Data } \\
\text { Collection }\end{array}$ Condition \\
\hline
\end{tabular}

$\begin{array}{cc} & \begin{array}{c}\text { To assess the } \\ \text { differences }\end{array} \\ \text { (Amarendran, } & \text { between using VC } \\ \text { George, } & \text { verses In-person } \\ \text { Gersappe, } & \text { assessment of } \\ \text { Krishnaswamy, } & \text { movement using } \\ \text { \& Warren, } & \text { the } \\ \text { 2011) } & \text { Abnormal } \\ \text { United States } & \text { Involuntary } \\ & \text { Movement Scale. }\end{array}$
Movement Scale.

\author{
Quasi-
experimental
Correlational/
Case-control
}

$$
\mathrm{N}=50
$$$$
\text { (male }=47 \text { ) }
$$

$\begin{array}{cc}\text { Dedicated } & \text { patients in the } \\ \text { VA system with }\end{array}$ equipment a history of with an ISDN antipsychotic connection. medications for at least 10 years.
Abnormal

Involuntary

Movement

Scale (AIMS).
Mental

Health
To evaluate VC use in a follow up clinic for patients

(Azad, Amos, Milne, \&

Power, 2012) Canada with memory

disorder

living in

rural areas.
To evaluate the use group VC to deliver a lifestyle intervention to

(Azar et al., 2015)

United States virtual small groups and to compare the change in body weight and BMI from baseline to 3 months.
(Chua, Craig, To compare VC to $\begin{array}{ll}\text { Wootton, \& } & \text { To compare VC } \\ \text { In person new }\end{array}$ $\begin{array}{cc}\text { Patterson, 2001) } & \text { In person new } \\ \text { patient neurology }\end{array}$ United Kingdom referrals

Descriptive
feasibility

$\mathrm{N}=50$ patients

Web based VC with mild to is assumed moderate because of memory reference to disorder "Video Link" in the article. without functional changes.

\section{Surveys \\ developed \\ by the study team.}

64 total (Men

ages $21-60$

BMI between

30 - 40, no

type 1

diabetes or

serious

medical

condition

or taking

weight loss

medication

or participating

in medically

supervised

weigh loss

program)

32 Intervention

32 control.
Demographics

via questionnaire

Height, Weight,

BP via

automated cuff.

Intervention:

Weight via

Bluetooth scale

weekly, attendance

at video visit,

self-monitoring

of body weight.

Obesity

Positive patient perceptions of VC. Measurements included: being understood by providers, having enough time, getting questions answered, and being the same as an in-person visit.

Participants in the intervention group lost significantly more weight, $3.5 \%$

(95\% CI 2.1\%,

$4.8 \%$ ), than

those randomized

to the control group. Participants attended 9 of 12

sessions on

average and weighed

themselves at

least once per week over the course of the intervention.

VC was less efficient and

Video newly referred $\begin{gathered}\text { In-person }=82 \text { medications } \\ \text { med }\end{gathered}$

RCT conferencing by PCP to non-urgent lines/SDN.

Neurologist visits in two hospital centers in the UK.

prescribed, and review of history, patient satisfaction, and diagnostic categories. not as well received by patients than In-person care. 


\section{Continued}

\begin{tabular}{|c|c|c|c|c|c|c|c|}
\hline $\begin{array}{c}\text { (Dorsey et al., } \\
\text { 2013) } \\
\text { United States }\end{array}$ & $\begin{array}{l}\text { To evaluate the } \\
\text { feasibility, } \\
\text { effectiveness, and } \\
\text { economic benefits } \\
\text { of VC care for } \\
\text { persons with } \\
\text { Parkinson disease } \\
\text { in their home. }\end{array}$ & RCT & $\begin{array}{c}\text { Web Based } \\
\text { VC }\end{array}$ & $\begin{array}{c}\mathrm{N}=20 \\
(\mathrm{VC}=9, \\
\text { control = 11) } \\
\text { patients with } \\
\text { Parkinson } \\
\text { disease and } \\
\text { Internet access } \\
\text { at home. }\end{array}$ & $\begin{array}{l}\text { Percentage of VC } \\
\text { visits completed as } \\
\text { scheduled, } \\
\text { Parkinson Disease } \\
\text { Questionnaire, } \\
\text { time, and travel. }\end{array}$ & Neurology & $\begin{array}{l}\text { VC offers similar } \\
\text { clinical control } \\
\text { and saved } \\
\text { participants } 100 \\
\text { miles of } \\
\text { travel and } 3 \\
\text { hours of time. }\end{array}$ \\
\hline $\begin{array}{l}\text { (Grady, 2002) } \\
\text { United States }\end{array}$ & $\begin{array}{l}\text { To compare the } \\
\text { costs to } \\
\text { patients, } \\
\text { medical } \\
\text { system, and } \\
\text { organization } \\
\text { of four } \\
\text { methods of mental } \\
\text { healthcare } \\
\text { in military } \\
\text { medical clinics. }\end{array}$ & Cost analysis & $\begin{array}{c}\text { Dedicated } \\
\text { equipment } \\
\text { with an ISDN } \\
\text { connection. }\end{array}$ & Not stated & Cost analysis & $\begin{array}{l}\text { Mental } \\
\text { Health }\end{array}$ & $\begin{array}{c}\text { The least } \\
\text { expensive method } \\
\text { of mental } \\
\text { healthcare } \\
\text { delivery was } \\
\text { tele-mental } \\
\text { health care } \\
\text { using video } \\
\text { conferencing. }\end{array}$ \\
\hline $\begin{array}{c}\text { (Grady \& } \\
\text { Melcer, 2005) } \\
\text { United States }\end{array}$ & $\begin{array}{l}\text { Compare treatment } \\
\text { and outcomes of } \\
\text { mental health care } \\
\text { via VC to in-person } \\
\text { care. }\end{array}$ & $\begin{array}{l}\text { Retrospective } \\
\text { chart review }\end{array}$ & $\begin{array}{c}\text { Video } \\
\text { conferencing } \\
\text { via phone } \\
\text { lines/ISDN. }\end{array}$ & $\begin{array}{c}\mathrm{N}=81(\mathrm{VC}= \\
51 \text { and } \\
\text { in-person = 30) } \\
\text { Adult patients } \\
\text { in the VA } \\
\text { system Seeking } \\
\text { mental health } \\
\text { care between } \\
\text { April 1, 1999 to } \\
\text { March 31, } \\
2000 .\end{array}$ & $\begin{array}{l}\text { The Global } \\
\text { Assessment } \\
\text { of Functioning } \\
\text { scale, Laboratory } \\
\text { studies, } \\
\text { number of } \\
\text { medications, } \\
\text { compliance, } \\
\text { mental status } \\
\text { examination, } \\
\text { recommendations } \\
\text { to utilize } \\
\text { resources, and } \\
\text { general number } \\
\text { and type } \\
\text { of diagnosis, } \\
\text { behavioral } \\
\text { characteristics of } \\
\text { the psychiatrists. }\end{array}$ & $\begin{array}{l}\text { Mental } \\
\text { Health }\end{array}$ & $\begin{array}{l}\text { Global assessment } \\
\text { of functioning and } \\
\text { compliance was } \\
\text { statistically } \\
\text { significant for } \\
\text { improved for the } \\
\text { VC group as } \\
\text { compared to } \\
\text { in-person } \\
\text { interactions. No } \\
\text { significant } \\
\text { differences in } \\
\text { number of tests, } \\
\text { self-help } \\
\text { recommendations, } \\
\text { assessments or } \\
\text { numbers of } \\
\text { medications } \\
\text { were seen. }\end{array}$ \\
\hline $\begin{array}{c}\text { (Hailey, 2008) } \\
\text { Canada }\end{array}$ & $\begin{array}{l}\text { To review evidence } \\
\text { related to } \\
\text { clinical and } \\
\text { administrative } \\
\text { outcome of } \\
\text { tele-mental health } \\
\text { studies. }\end{array}$ & Review article & $\begin{array}{l}\text { Specific VC } \\
\text { type for each } \\
\text { article Not } \\
\text { discussed. }\end{array}$ & $\begin{array}{l}72 \text { published } \\
\text { papers } \\
\text { "Conducted } \\
\text { in a } \\
\text { scientifically } \\
\text { valid } \\
\text { Manner" } \\
\text { reporting } \\
\text { clinical or } \\
\text { administrative } \\
\text { outcomes } \\
\text { controlled } \\
\text { studies } \\
\text { VC was } \\
\text { compared with } \\
\text { a non-VC } \\
\text { alternative and } \\
\text { Non-controlled } \\
\text { studies } 20 \text { or } \\
\text { more } \\
\text { subjects related } \\
\text { to tele-mental } \\
\text { health. }\end{array}$ & $\begin{array}{l}\text { General mental } \\
\text { health, depression, } \\
\text { panic disorder, } \\
\text { smoking, cognitive } \\
\text { disability, } \\
\text { pediatrics, OCD, } \\
\text { schizophrenia, } \\
\text { substance abuse, } \\
\text { eating disorders, } \\
\text { suicide prevention, } \\
\text { PTSD. } \\
\end{array}$ & $\begin{array}{l}\text { Mental } \\
\text { Health }\end{array}$ & $\begin{array}{l}\text { The quality of VC } \\
\text { studies was } \\
\text { limited with most } \\
\text { being preliminary. } \\
\text { The two RCTs in } \\
\text { the paper found no } \\
\text { difference in } \\
\text { quality of VC } \\
\text { verses In-person } \\
\text { and one } \\
\text { non-random study } \\
\text { found improved } \\
\text { mental health } \\
\text { outcomes for VC } \\
\text { compared to } \\
\text { In-person } \\
\text { encounters. }\end{array}$ \\
\hline
\end{tabular}




\section{Continued}

\begin{tabular}{|c|c|c|c|c|c|c|c|}
\hline $\begin{array}{l}\text { (Khatri, } \\
\text { Marziali, } \\
\text { Tchernikov, } \\
\text { \& Shepherd, } \\
\text { 2014) } \\
\text { Canada }\end{array}$ & $\begin{array}{l}\text { To compare the } \\
\text { provision and } \\
\text { outcomes of group } \\
\text { cognitive } \\
\text { behavioral therapy } \\
\text { when delivered } \\
\text { using VC as } \\
\text { compared to } \\
\text { in-person } \\
\text { delivery. }\end{array}$ & $\begin{array}{l}\text { Non-randomized } \\
\text { Pre/post mixed } \\
\text { methods quasi } \\
\text { experimental } \\
\text { (Allowed } \\
\text { participants to } \\
\text { choose VC or } \\
\text { In-person). }\end{array}$ & $\begin{array}{l}\text { Web based } \\
\text { VC }\end{array}$ & $\begin{array}{c}\mathrm{N}=18 \text { adults } \\
(8=\mathrm{VC}, 10= \\
\text { In-person) with } \\
\text { diagnosis of } \\
\text { mood, anxiety } \\
\text { disorder, and/or } \\
\text { adjustment } \\
\text { disorder with } \\
\text { access to a } \\
\text { computer, } \\
\text { webcam, and } \\
\text { internet. }\end{array}$ & $\begin{array}{l}\text { Beck Depression } \\
\text { Inventory Second } \\
\text { Edition and } \\
\text { Qualitative theme } \\
\text { analysis }\end{array}$ & $\begin{array}{l}\text { Mental } \\
\text { Health }\end{array}$ & $\begin{array}{l}\text { BDI-II scores and } \\
\text { qualitative } \\
\text { analysis of the } \\
\text { themes were } \\
\text { similar across the } \\
\text { two delivery } \\
\text { formats. VC is } \\
\text { comparable to } \\
\text { in-person group } \\
\text { based cognitive } \\
\text { therapy. }\end{array}$ \\
\hline $\begin{array}{l}\text { (Kitamura, } \\
\text { Zurawel-Balaur } \\
\text { a, \& Wong, } \\
\text { 2010) } \\
\text { Canada }\end{array}$ & $\begin{array}{l}\text { Use systematic } \\
\text { review of the } \\
\text { literature to } \\
\text { evaluate the } \\
\text { feasibility of } \\
\text { assessing, } \\
\text { monitoring, and } \\
\text { managing oncology } \\
\text { patients via video } \\
\text { conferencing. }\end{array}$ & $\begin{array}{l}\text { Systematic } \\
\text { review }\end{array}$ & $\begin{array}{c}\text { Specific VC } \\
\text { type for each } \\
\text { article Not } \\
\text { discussed. }\end{array}$ & $\begin{array}{c}\mathrm{N}=19 \\
\text { published } \\
\text { articles of } 15 \\
\text { clinical } \\
\text { oncology } \\
\text { patient groups: } \\
\text { one small } \\
\text { RCT; } 7 \\
\text { non-randomized } \\
\text { with control } \\
\text { groups, and } 7 \\
\text { case studies. } \\
\text { Total } 709 \text { VC } \\
\text { study patients } \\
\text { and } 346 \text { control } \\
\text { patients. }\end{array}$ & $\begin{array}{l}\text { Reported outcomes } \\
\text { included patient } \\
\text { satisfaction (no } \\
\text { validated scales) } \\
\text { and preference for } \\
\text { VC consultation, } \\
\text { costs, provider } \\
\text { satisfaction and } \\
\text { convenience. } \\
\text { accessibility of } \\
\text { care and clinical } \\
\text { outcomes limited. }\end{array}$ & Oncology & $\begin{array}{l}\text { VC is feasible, } \\
\text { effective for } \\
\text { assessing, } \\
\text { monitoring, and } \\
\text { managing } \\
\text { oncology patients, } \\
\text { and clinical } \\
\text { outcomes were } \\
\text { not compromised; } \\
\text { time and cost were } \\
\text { comparable or } \\
\text { reduced. Limited } \\
\text { power of inference } \\
\text { with small } \\
\text { samples and } \\
\text { methodological } \\
\text { weaknesses. }\end{array}$ \\
\hline $\begin{array}{l}\text { (Lewis, 2003) } \\
\text { United States }\end{array}$ & $\begin{array}{l}\text { To evaluate a web } \\
\text { monitoring system } \\
\text { intended to } \\
\text { improve } \\
\text { walking ability } \\
\text { post-stroke. }\end{array}$ & Case-Study & $\begin{array}{l}\text { Web based VC } \\
\text { system with } \\
\text { integrated } \\
\text { performance } \\
\text { indicators. }\end{array}$ & $\mathrm{N}=2$ & $\begin{array}{c}\text { Post-satisfaction } \\
\text { questionnaire. }\end{array}$ & Rehabilitation & $\begin{array}{l}\text { One participant } \\
\text { evaluated the } \\
\text { system favorable } \\
\text { and one } \\
\text { participant wanted } \\
\text { an in person } \\
\text { therapist. }\end{array}$ \\
\hline $\begin{array}{c}\text { (Lipman, } \\
\text { Kenny, \& } \\
\text { Marziali, 2011) } \\
\text { Canada }\end{array}$ & $\begin{array}{l}\text { To evaluate the } \\
\text { feasibility of } \\
\text { providing } \\
\text { web-based } \\
\text { support and } \\
\text { education for } \\
\text { single mothers. }\end{array}$ & $\begin{array}{l}\text { Pre/post mixed } \\
\text { methods } \\
\text { descriptive } \\
\text { quasi } \\
\text { experimental. }\end{array}$ & $\begin{array}{c}\text { Web Based } \\
\text { VC }\end{array}$ & $\begin{array}{c}\mathrm{N}=15 \text { single } \\
\text { mothers with } \\
\text { health disparity } \\
\text { having children } \\
\text { ages } 3 \text { - } 9 .\end{array}$ & $\begin{array}{c}\text { Qualitative } \\
\text { interviews, } \\
\text { demographic, } \\
\text { medications, } \\
\text { CES-D, } \\
\text { Rosenberg } \\
\text { Self-Esteem } \\
\text { Scale, Social } \\
\text { Provisions } \\
\text { Scale, and } \\
\text { Parenting Stress }\end{array}$ & $\begin{array}{l}\text { Mental } \\
\text { Health }\end{array}$ & $\begin{array}{c}\text { Positive } \\
\text { perceptions of } \\
\text { the VC } \\
\text { intervention via } \\
\text { qualitative } \\
\text { evaluation and } \\
\text { improvement } \\
\text { of all quantitative } \\
\text { outcome } \\
\text { measures. }\end{array}$ \\
\hline
\end{tabular}

Comparing the effectiveness of online VC with

(Man, Soong, interactive Tam, \& software, Hui-Chan, interactive software 2005) alone, in-person

Hong Kong, and control for China problem-solving skill training groups for persons with Brain Injury.
Comparative

effectiveness

Pre/post quasi

experimental



VC with therapistadministered group was effective in improving problem solving skills in persons with ABI. 


\section{Continued}

\begin{tabular}{|c|c|c|c|c|c|c|c|}
\hline $\begin{array}{l}\text { (Marhefka } \\
\text { et al., 2013) } \\
\text { United States }\end{array}$ & $\begin{array}{l}\text { To evaluate } \\
\text { participant } \\
\text { satisfaction, } \\
\text { facilitators, } \\
\text { experiences and } \\
\text { technology of } \\
\text { video group } \\
\text { delivery of the } \\
\text { Healthy } \\
\text { Relationships } \\
\text { intervention for } \\
\text { women living } \\
\text { with HIV. }\end{array}$ & Qualitative & $\begin{array}{l}\text { Video } \\
\text { education } \\
\text { using a } \\
\text { terminal at } \\
\text { primary care } \\
\text { clinics to } \\
\text { connect to a } \\
\text { remote } \\
\text { education } \\
\text { group. }\end{array}$ & $\mathrm{N}=4$ & $\begin{array}{l}\text { Demographics, } \\
\text { qualitative } \\
\text { discussion and } \\
\text { open-ended } \\
\text { questionnaire. }\end{array}$ & HIV & $\begin{array}{l}\text { Video group } \\
\text { participation was } \\
\text { feasible and } \\
\text { valued by } \\
\text { participants. } \\
\text { Efficacy was not } \\
\text { evaluated. }\end{array}$ \\
\hline $\begin{array}{c}\text { (Martin-Khan et } \\
\text { al., 2012) } \\
\text { Australia }\end{array}$ & $\begin{array}{l}\text { Evaluating the use } \\
\text { of VC versus } \\
\text { standard in-person } \\
\text { care to establish a } \\
\text { diagnosis of } \\
\text { dementia. }\end{array}$ & RCT & $\begin{array}{l}\text { VC with ISDN } \\
\text { connection. }\end{array}$ & $\begin{array}{c}\mathrm{N}=205 \\
(\mathrm{VC}=100 \\
\text { In-person = } \\
\text { 105) patients } \\
\text { aged } 50 \text { or } \\
\text { older referred } \\
\text { for cognitive } \\
\text { assessment. }\end{array}$ & $\begin{array}{c}\text { Mini-Mental State } \\
\text { Examination } \\
\text { (MMSE), Rowland } \\
\text { Universal } \\
\text { Dementia } \\
\text { Assessment Scale } \\
\text { (RUDAS), Clock } \\
\text { Face Test (CFT), } \\
\text { Letter Naming } \\
\text { Verbal Fluency } \\
\text { Test (FAS), } \\
\text { Naming Animals } \\
\text { Verbal Fluency, } \\
\text { Geriatric } \\
\text { Depression } \\
\text { Scale-15 questions } \\
\text { (GDS-15), } \\
\text { Informant } \\
\text { Questionnaire on } \\
\text { Cognitive Decline } \\
\text { in the Elderly } \\
\text { (IQCODE), } \\
\text { Neuropsychiatric } \\
\text { Inventory Short } \\
\text { form (NPI-Q), and } \\
\text { Disability. } \\
\text { Assessment for } \\
\text { Dementia (DAD). }\end{array}$ & $\begin{array}{l}\text { Mental } \\
\text { Health }\end{array}$ & $\begin{array}{c}\text { In general, there } \\
\text { was agreement } \\
\text { between the VC } \\
\text { and in-person } \\
\text { assessments with } \\
\text { only } 1 \% \\
\text { difference } \\
\text { between the } \\
\text { total scores for } \\
\text { overall } \\
\text { agreement. }\end{array}$ \\
\hline $\begin{array}{c}\text { (Marziali \& } \\
\text { Donahue, 2006) } \\
\text { Canada }\end{array}$ & $\begin{array}{l}\text { To compare the } \\
\text { effectiveness of an } \\
\text { internet based VC } \\
\text { support } \\
\text { intervention } \\
\text { to a no } \\
\text { treatment group } \\
\text { in a sample } \\
\text { of caregivers of } \\
\text { older adults with } \\
\text { neurodegenerative } \\
\text { disease. }\end{array}$ & RCT & $\begin{array}{l}\text { Web Based } \\
\text { VC }\end{array}$ & $\begin{array}{c}\mathrm{N}=66 \\
\text { caregivers of } \\
\text { older adults } \\
\text { with } \\
\text { Alzheimer's, } \\
\text { stroke related } \\
\text { dementia, and } \\
\text { Parkinson's. }\end{array}$ & $\begin{array}{l}\text { Health Status } \\
\text { Questionnaire 12, } \\
\text { abbreviated } \\
\text { Medical } \\
\text { Outcomes Study } \\
\text { 36, Center for } \\
\text { Epidemiologic } \\
\text { Studies-Depression } \\
\text { scale, self-report } \\
\text { of depressive } \\
\text { affect and } \\
\text { behavior, } \\
\text { instrumental } \\
\text { ADLs, Revised } \\
\text { Memory and } \\
\text { Behavior Problems } \\
\text { Checklist, and } \\
\text { Multi-dimensional } \\
\text { Scale of Perceived } \\
\text { Social Support. }\end{array}$ & $\begin{array}{c}\text { Neuro/ } \\
\text { Mental } \\
\text { Health/ } \\
\text { Caregivers }\end{array}$ & $\begin{array}{l}\text { Over half of the } \\
\text { caregivers had } \\
\text { never used } \\
\text { computers but } \\
\text { reported that the } \\
\text { training was } \\
\text { sufficient and 78\% } \\
\text { indicated the } \\
\text { website was easy to } \\
\text { use. } 95 \% \text { rated } \\
\text { using the } \\
\text { computers as a } \\
\text { positive experience. } \\
\text { At least one } \\
\text { participant } \\
\text { reported that VC } \\
\text { was more helpful } \\
\text { than in-person. The } \\
\text { VC group improved } \\
\text { on reported stress- } \\
\text { and the control } \\
\text { group worsened. } \\
\text { There was } \\
\text { significantly } \\
\text { higher attrition in } \\
\text { the control group. }\end{array}$ \\
\hline
\end{tabular}




\section{Continued}

\begin{tabular}{|c|c|c|c|c|c|c|c|}
\hline $\begin{array}{l}\text { (Marziali \& } \\
\text { Garcia, 2011) } \\
\text { Canada }\end{array}$ & $\begin{array}{c}\text { To compare } \\
\text { dementia } \\
\text { caregivers' stress } \\
\text { and health status } \\
\text { when enrolled in } \\
\text { one of two groups: } \\
\text { internet chat } \\
\text { support that } \\
\text { included caregiver } \\
\text { handbook and } 6 \\
\text { videos on } \\
\text { managing } \\
\text { caregiving versus } \\
\text { Internet based VC } \\
\text { delivered by a } \\
\text { clinician with } \\
\text { access to caregiver } \\
\text { handbook, support. }\end{array}$ & $\begin{array}{l}\text { Non-randomized } \\
\text { Comparative } \\
\text { effectiveness } \\
\text { pre/post design } \\
\text { quasi } \\
\text { experimental. }\end{array}$ & $\begin{array}{l}\text { Web Based } \\
\text { VC }\end{array}$ & $\begin{array}{l}\mathrm{N}=91 \text { (Chat } \\
\text { Group N = 40; } \\
\text { Video Group } \\
\mathrm{N}=51) .\end{array}$ & $\begin{array}{l}\text { Demographic } \\
\text { information, } \\
\text { Eysenck } \\
\text { Personality } \\
\text { Questionnaire- } \\
\text { Revised, } \\
\text { neuroticism, } \\
\text { Revised Scale } \\
\text { for Caregiver } \\
\text { Self-efficacy, } \\
\text { beliefs about } \\
\text { caregiving, } \\
\text { Perceived } \\
\text { Social } \\
\text { Support, } \\
\text { Health Status } \\
\text { Questionnaire, } \\
\text { Center for } \\
\text { Epidemiologic } \\
\text { Studies } \\
\text { Depression } \\
\text { Scale, } \\
\text { Functional } \\
\text { Autonomy } \\
\text { Measurement } \\
\text { System, current } \\
\text { outside service } \\
\text { use, and intent } \\
\text { to continue } \\
\text { caregiving } \\
\text { at home. }\end{array}$ & $\begin{array}{c}\text { Mental } \\
\text { Health/ } \\
\text { Caregivers }\end{array}$ & $\begin{array}{l}\text { Both groups } \\
\text { significantly } \\
\text { improved in } \\
\text { self-efficacy. } \\
\text { Neither group } \\
\text { changed in use of } \\
\text { health and social } \\
\text { services. } \\
\text { When compared, } \\
\text { the VC group had } \\
\text { greater } \\
\text { improvement in } \\
\text { mental health and } \\
\text { reported distress } \\
\text { scores but the Chat } \\
\text { group had lower } \\
\text { distress reported } \\
\text { for managing } \\
\text { Instrumental } \\
\text { Activities of Daily } \\
\text { Living (IADLs). }\end{array}$ \\
\hline $\begin{array}{c}\text { (Norman, 2006) } \\
\text { United } \\
\text { Kingdom }\end{array}$ & $\begin{array}{l}\text { To review the } \\
\text { evidence related to } \\
\text { the use of VC for } \\
\text { mental health } \\
\text { issues in the } \\
\text { United Kingdom. }\end{array}$ & Review article & $\begin{array}{l}\text { Specific VC } \\
\text { type for each } \\
\text { article Not } \\
\text { discussed }\end{array}$ & 72 Abstracts & $\begin{array}{c}\text { Efficacy, } \\
\text { cost-effectiveness, } \\
\text { and satisfaction. }\end{array}$ & $\begin{array}{l}\text { Mental } \\
\text { Health }\end{array}$ & $\begin{array}{l}\text { VC has been cost } \\
\text { effective and } \\
\text { reliable method for } \\
\text { patients with } \\
\text { mental health } \\
\text { issues. Limitations } \\
\text { still exist that need } \\
\text { to be addressed } \\
\text { including type of } \\
\text { patients and } \\
\text { confidentiality. }\end{array}$ \\
\hline $\begin{array}{l}\text { (Peel, Russell, } \\
\text { \& Gray, 2011) } \\
\text { Australia }\end{array}$ & $\begin{array}{l}\text { To evaluate an } \\
\text { in-home VC } \\
\text { system called } \\
\text { eHAB for } \\
\text { feasibility of } \\
\text { home } \\
\text { rehabilitation to } \\
\text { older adults. }\end{array}$ & Feasibility & $\begin{array}{l}\text { Web based VC } \\
\text { system }\end{array}$ & $\mathrm{N}=44$ & $\begin{array}{l}\text { No actual } \\
\text { participants were } \\
\text { recruited and } \\
\text { monitored using } \\
\text { the VC system. }\end{array}$ & Rehabilitation & $\begin{array}{l}\text { This VC system } \\
\text { was not feasible in } \\
\text { an older population } \\
\text { with rehabilitation } \\
\text { needs. Special } \\
\text { needs of this } \\
\text { population } \\
\text { require an easy } \\
\text { to use light and } \\
\text { mobile system } \\
\text { or in home } \\
\text { support to operate } \\
\text { the equipment. } \\
\text { The unique needs } \\
\text { including } \\
\text { decreased vision, } \\
\text { hearing, and } \\
\text { decreased } \\
\text { physical mobility } \\
\text { need to } \\
\text { be addressed. }\end{array}$ \\
\hline
\end{tabular}




\section{Continued}

\begin{tabular}{|c|c|c|c|c|c|c|c|}
\hline $\begin{array}{c}\text { (Somers et al., } \\
\text { 2015) } \\
\text { United States }\end{array}$ & $\begin{array}{l}\text { Using VC on a } \\
\text { tablet computer } \\
\text { to deliver a brief } \\
\text { Pain Coping } \\
\text { Skills Training } \\
\text { (PCST) for } \\
\text { patients with } \\
\text { persistent pain } \\
\text { from cancer. }\end{array}$ & $\begin{array}{c}\text { Pre/post design } \\
\text { for feasibility } \\
\text { and } \\
\text { acceptability } \\
\text { quasi } \\
\text { experimental. }\end{array}$ & $\begin{array}{c}\text { Web Based VC } \\
\text { (Skype) } \\
\text { on a tablet } \\
\text { computer. }\end{array}$ & $\begin{array}{c}6 \text { male and } 19 \\
\text { female patients } \\
\text { with cancer; } \\
\text { mean age of } \\
53.9+12.6 \text { yrs. }\end{array}$ & $\begin{array}{l}\text { Measures included } \\
\text { pain, physical } \\
\text { functioning and } \\
\text { symptoms, } \\
\text { psychological } \\
\text { distress, } \\
\text { self-efficacy } \\
\text { for pain } \\
\text { management } \\
\text { and pain } \\
\text { catastrophizing } \\
\text { via pre-and } \\
\text { post-intervention } \\
\text { questionnaires. } \\
\text { collected on a } \\
\text { secure web-site } \\
\text { via the } \\
\text { mobile tablet. }\end{array}$ & Oncology & $\begin{array}{l}18 \text { of the } 25 \\
\text { participants } \\
\text { completed all } 4 \\
\text { sessions and } 1 \\
\text { completed } 3 \\
\text { sessions with } \\
\text { post-intervention } \\
\text { outcome data for } \\
\text { the 19; video } \\
\text { conferencing was } \\
\text { feasible and } \\
\text { acceptable. } \\
\text { Pre-post } \\
\text { interventions } \\
\text { scores showed } \\
\text { significantly } \\
\text { decreased pain } \\
\text { severity, physical } \\
\text { symptoms, } \\
\text { psychological } \\
\text { distress, pain } \\
\text { catastrophizing. } \\
\text { Limited } \\
\text { generalizability } \\
\text { with small, } \\
\text { non-randomized } \\
\text { samples. }\end{array}$ \\
\hline $\begin{array}{l}\text { (Temple, } \\
\text { Drummond, } \\
\text { Valiquette, \& } \\
\text { Jozsvai, 2010) } \\
\text { Canada }\end{array}$ & $\begin{array}{l}\text { To compare } \\
\text { assessment of } \\
\text { persons with } \\
\text { intellectual } \\
\text { disability (ID) } \\
\text { using VC to in } \\
\text { person } \\
\text { assessments. }\end{array}$ & $\begin{array}{c}\text { Descriptive } \\
\text { observational }\end{array}$ & $\begin{array}{l}\text { Encrypted web } \\
\text { based VC }\end{array}$ & $\begin{array}{c}\mathrm{N}=19 \text { adults } \\
\text { (23 - 63) with } \\
\text { Intellectual } \\
\text { disability. }\end{array}$ & $\begin{array}{c}\text { Wechsler } \\
\text { Abbreviated Scale } \\
\text { of Intelligence } \\
\text { and the } \\
\text { Beery-Buktenica } \\
\text { Developmental } \\
\text { Test of } \\
\text { Visual-Motor } \\
\text { Integration-IV. }\end{array}$ & $\begin{array}{l}\text { Mental } \\
\text { Health }\end{array}$ & $\begin{array}{l}\text { There are no } \\
\text { statistically } \\
\text { significant } \\
\text { differences } \\
\text { between } \\
\text { assessment of ID } \\
\text { between VC and } \\
\text { in-person } \\
\text { assessment. }\end{array}$ \\
\hline $\begin{array}{c}\text { (Vadheim et al., } \\
\text { 2010) } \\
\text { United States }\end{array}$ & $\begin{array}{l}\text { To assess the } \\
\text { feasibility of } \\
\text { delivering a } \\
\text { Diabetes } \\
\text { Prevention } \\
\text { Program group } \\
\text { intervention } \\
\text { through VC versus } \\
\text { In-person. }\end{array}$ & $\begin{array}{l}\text { Descriptive/ } \\
\text { Case-control }\end{array}$ & Not described & $\mathrm{N}=19$ & $\begin{array}{l}\text { Attendance, } \\
\text { completion, } \\
\text { weight, blood } \\
\text { glucose, lipid } \\
\text { values, current } \\
\text { medication, } \\
\text { self-monitoring, } \\
\text { dietary intake. }\end{array}$ & Diabetes & $\begin{array}{l}\text { All participants } \\
\text { completed the } \\
\text { In-person group } \\
\text { and } 88 \% \text { completed } \\
\text { the telehealth } \\
\text { group. All } \\
\text { participants } \\
\text { mproved } \\
\text { biophysical } \\
\text { measurements and } \\
\text { there was not } \\
\text { statistical } \\
\text { difference } \\
\text { between the VC } \\
\text { and In-person } \\
\text { group. }\end{array}$ \\
\hline $\begin{array}{l}\text { (Wakefield, } \\
\text { Buresh, } \\
\text { Flanagan, \& } \\
\text { Kienzle, 2004) } \\
\text { United States }\end{array}$ & $\begin{array}{c}\text { To assess } \\
\text { satisfaction and } \\
\text { outcomes of VC } \\
\text { for specialty care } \\
\text { for residents of a } \\
\text { long-term care } \\
\text { center. }\end{array}$ & Descriptive & $\begin{array}{c}\text { VC with ISDN } \\
\text { connection } \\
\text { provided by the } \\
\text { Iowa } \\
\text { Communications } \\
\text { Network. }\end{array}$ & $\begin{array}{c}\mathrm{N}=76 \text { patients } \\
\text { living in a } \\
\text { nursing home } \\
\text { and needing a } \\
\text { specialty } \\
\text { medical } \\
\text { consultation } \\
\text { appointment. }\end{array}$ & $\begin{array}{l}\text { Outcomes (Change } \\
\text { in treatment yet } \\
\text { remaining at the } \\
\text { care facility, no } \\
\text { change in } \\
\text { treatment and } \\
\text { remain at care } \\
\text { facility, other), } \\
\text { Satisfaction with } \\
\text { VC. }\end{array}$ & $\begin{array}{c}\text { Long-term } \\
\text { care }\end{array}$ & $\begin{array}{l}\text { There was a high } \\
\text { level of satisfaction } \\
\text { for both patients } \\
\text { and providers. VC } \\
\text { allowed most } \\
\text { patients to remain } \\
\text { in the long-term } \\
\text { care facility instead } \\
\text { of having to leave } \\
\text { for specialty } \\
\text { appointment. }\end{array}$ \\
\hline
\end{tabular}




\section{Continued}

(Wong,

Martin-Khan, To validate the

Rowland, RUDAS dementia

Varghese, \& screening via video

Gray, 2011) conferencing.

Australia
RCT-this

To assess patient article

and provider

Weiner et al., satisfaction with

United States unscheduled VC

for persons living

in a Nursing home. interventio group.

presents

early

findings

from

group.

$\begin{array}{cc}\text { Modem Web Based } & \mathrm{N}=187 \\ \text { VC } & \text { patients living } \\ \text { in a nursing } \\ \text { home. }\end{array}$

Patient

characteristics, Long-term reason for VC, satisfaction. cong-term
care

Long-term
care

Medical decision-making was easier via $\mathrm{VC}$ verses phone consultation. No patient reported that VC communication was different than usual care.

There is no statistically significant difference in mean RUDAS scores for in-person or Video Conference administered assessments at both the total score, and individual domain levels. Hence the RUDAS can be reliability administered and scored via Video conference.

$\mathrm{VC}$ in combination with other home monitoring was easy to use and had high satisfaction; outcomes for

patient recall data documented

reduced number of hospital

readmissions \&

days in hospital for patients with angina, and improved quality
of life and FS for both groups: HF and angina. No significant differences in physician visits beween VC and usual care groups. The type of monitor is not reported nor the \% of time that VC transmission problems resulted in telephone interviews only. 


\section{Continued}

\begin{tabular}{|c|c|c|c|c|c|c|c|}
\hline $\begin{array}{c}\text { (Woolf et al., } \\
\text { 2015) } \\
\text { United } \\
\text { Kingdom }\end{array}$ & $\begin{array}{l}\text { Assess the } \\
\text { feasibility for } \\
\text { comparing remote } \\
\text { therapies for word } \\
\text { finding for persons } \\
\text { with aphasia in four } \\
\text { groups; two remote } \\
\text { sites (University } \\
\text { and Clinical), } \\
\text { in-person therapy, } \\
\text { and a attention } \\
\text { control. }\end{array}$ & Feasibility & $\begin{array}{c}\text { Web Based } \\
\text { VC }\end{array}$ & $\begin{array}{c}\mathrm{N}=21 \text { people } \\
\text { with aphasia } \\
\text { after left } \\
\text { hemisphere } \\
\text { stroke. }\end{array}$ & $\begin{array}{c}\text { Feasibility and } \\
\text { word retrieval via } \\
\text { picture naming and } \\
\text { conversation. }\end{array}$ & $\begin{array}{l}\text { Neurology/ } \\
\text { Stroke }\end{array}$ & $\begin{array}{l}\text { Participants gave } \\
\text { good ratings for } \\
\text { connectivity and } \\
\text { for visual and } \\
\text { sound quality. } \\
\text { They } \\
\text { problem-solved } \\
\text { when needed by } \\
\text { moving the } \\
\text { equipment or } \\
\text { redialing. } \\
\text { Participants in the } \\
\text { therapy groups } \\
\text { reported } \\
\text { independently } \\
\text { practicing. } \\
\text { Compliance and } \\
\text { Participants in all } \\
\text { groups improved } \\
\text { but those who } \\
\text { received remote } \\
\text { therapy from the } \\
\text { clinical site were } \\
\text { most improved. }\end{array}$ \\
\hline
\end{tabular}

The studies that found VC to be ineffective attributed the lack of efficacy to the age of the participants, technical difficulty, or a desire to have in-person sessions. A study of real-time web-based monitoring on two patients recovering from strokes was done to help improve their walking ability. One individual strongly favored the technology and the other strongly opposed it due to wanting the primary therapist in person at all times [53]. A study on in-home video conferencing for geriatric rehabilitation was not successful for a variety of reasons including vision and hearing impairment, client anxiety and stress, dementia, cluttered home environment and in-home assistance being needed to operate the equipment and the need to remain with the patient due to high risk for falls [54]. The authors of this study recommended use of video conferencing in a geriatric population that is higher functioning with fewer co-morbidities. All of these studies had limitations of very small sample sizes. Video conferencing should be tested with larger sample sizes to effectively determine its utility.

\subsection{Mental Health}

Most of the literature on use of VC is related to mental health and neurological disorders. Ten different studies report the use of VC in the care of patients and families with dementia, neurodegenerative disorders, and mental health issues [33]-[35] [38] [41] [43]-[45] [48] [52]. Most of these studies had positive results, including being more cost effective and time efficient for both the healthcare provider and patient [34] [35]. However, one study had mixed results. Chua (2001) compared in-person neurological referrals to VC neurological referrals. The VC group had an increased number of assessments, there were no differences in the number of medications prescribed and patients were satisfied with both types of consultation. However, some participants reported embarrassment related to being on camera [33].

\subsection{Mental Health Diagnosis \& Assessment}

Diagnosis and assessment related to mental health and neurological disorders is possible using VC. Amarendran et al. conducted a study on the ability to complete an assessment of involuntary movement using VC compared to an in-person assessment for 50 participants who had been exposed to antipsychotic medications for a minimum of 10 years [30]. The measure used was the Abnormal Involuntary Movement Scale (AIMS) and the assessment was conducted with two raters in the room and two other raters watching from a nearby remote location; the raters were rotated for each session. An analysis was conducted using multilevel modeling to assess the extent to which total variance in rating was due to the differences between raters rating the same patient and differences between patient to patient. The mean Global Rating for the in-person assessment was $1.35(\mathrm{sd}=1.03)$ 
and the mean Global Rating for the VC assessment was 1.37 ( $\mathrm{sd}=0.92)$. The difference was not significant.

Martin-Khan (2012) evaluated the use of VC for establishing the diagnosis of dementia and reported that in 205 adults (aged 50 or older), the clinical assessment for the diagnosis of dementia using valid and reliable instruments can be accomplished using VC. During intellectual assessments, results show there is no statistically significant difference in test results compared to in-person care [48]. Another study [41] evaluated VC compared to three other interventions: computer assisted treatment, in-person treatment, and no treatment for 103 patients with Acquired Brain Injury (ABI). While the in-person administered training showed better improvements in self-efficacy and in problem-solving, the diagnosis of dementia using VC was feasible, effective and there were no differences in other measures. Thus, a combination of in-person and VC training for patients with ABI may be beneficial.

Another study found that VC and in-person assessment did differ between groups. Temple et al. conducted a descriptive observational study on the use of video conferencing (VC) compared to in-person assessments of persons with intellectual disabilities (ID) [48]. There were 19 participants with a mean age of 38.5 and some form of ID along with no visual or hearing impairment. The participants were assessed using two measures, the Wechsler Abbreviated Scale of Intelligence (WASI) and the Beery-Buktenice Developmental test of visualmotor integration-IV (VMI). Participants had two testing sessions, one over VC and one in-person with a 5 to 21 month time in between with the mean time being 10.4 months. The results from the WASI indicated the visual IQ (VIQ) scores were higher in-person (in-person mean $=70$, VC mean $=67$ ). Though the performance IQ (PIQ) scores were higher for the VC group (VC mean $=68.4$, in-person mean $=66.3$ ). The full scale IQ (FSIQ) and VMI on average differed less than 1 point (FSIQ diff $=-0.8$, VMI diff $=-0.6$ ). Analyses show that the WASI scores for FSIQ and PIQ had no significant change. The change in the VIQ however was found to be significant and the VMI scores did not differ significantly. The results indicate that using VC to conduct intellectual assessments is effective; however the sub-scoring of the WASI, specifically the VIQ, suggests that this should be done cautiously. Informal questioning revealed that patients and providers both see VC as an acceptable method of performing assessments. However, this study was also limited by its small sample size.

\subsection{Mental Health Treatment}

Treatment of mental health and neurological disorders for both patients and caregivers can be delivered through or assisted by VC. A mixed methods study was conducted with 18 individuals diagnosed with mood and or anxiety disorder using a VC provided cognitive behavioral therapy (CBT) intervention program compared to in-person CBT therapy. The study showed support for use of VC in the provision of CBT compared to in-person CBT as there were no significant differences in the VC group compared to the in-person group [38].

Marzali \& Donahue (2006) compared the effectiveness of a VC support group to a no treatment usual care group in a sample of 66 caregivers of older adults with Alzheimer's disease. The study concluded that the caregivers rated the computer use as a positive experience and that the VC group improved on caregiver stress while the no treatment group reported increased stress. Subsequently, Marzali \& Garcia (2011) compared the reported stress and health status of caregivers of persons with dementia using two groups: an internet chat support group that included a caregiver handbook and six educational videos versus internet-based videoconferencing delivered by a clinician that also included the caregiver handbook. In this study of 91 caregivers, both groups improved on self-efficacy and neither group changed in their use of health and social services. However, the VC group had greater improvement in mental health and stress scores.

Grady et al. conducted a retrospective chart review to evaluate the effect of telehealth care for remote military populations suffering from mental health conditions [36]. The records reviewed came from two locations, the National Navel Medical Center and the Patuxent River Clinic. These locations were chosen because they were similarly staffed and provided similar access to medical services. The National Navel Medical Center has offered telehealth care for a few years and the Patuxent Clinic did not offer telehealth services, making the Patuxent Clinic an appropriate control. The principal measure being used was the Global Assessment of Functioning and the treatments measured were laboratory studies, number of medications used, adherence, mental status examinations, general number and type of diagnosis. Of the records available, 81 had two or more visits as required to evaluate a change in Global Assessment of Functioning. Thirty of the participants had in-person encounters and 51 had VC encounters. The in-person groups initial mean score was 56 and the final in-person score was 65 with a mean change being 8.4. The VC group's mean initial score was 54 and the final was 69 , 
with a mean change in being 15.3. The change between VC and in-person groups was found to be significant. The VC group also had significantly more patients who demonstrated adherence (used medications and came to consultation appointments) than the in-person group ( $\mathrm{VC}=94 \%$, in-person $=89 \%$ ). It was also determined that the time between appointments was significantly shorter in the VC group with $73 \%$ of the appointments within 30 days as compared to $62 \%$ within the same time frame in the in-person group.

Grady, 2002, conducted a comparative cost analysis between the currently available methods of providing treatment of mental health issues for military personnel and veterans [35]. The four options examined were using the locally available heath care providers, travel to a military medical center, travel to a local medical facility where there is a circuit riding provider, and using VC to see a remotely located heath care provider. The cost of each service was calculated taking into account the cost to the patient, cost to the military, and cost to the clinic/provider. These costs included travel, missed work/lost time, equipment cost, reimbursement, and operational cost. The total monthly cost calculated revealed that the most expensive was travel to a military medical facility (\$6986.72), followed by using the local network of health care providers (\$5510.39), then traveling to a local facility where a circuit provider is located $(\$ 5421.67)$ then using VC systems $(\$ 4599.73)$. The primary reason for the reduced cost of telehealth is the estimated reduction in hospitalization costs $(\$ 648.50,14 \%)$ along with reduced appointment times, 5 - 10 min, and the removal of much of the travel time for both the patient and provider. The initial installation of a VC system is estimated at $\$ 719.71$ per month but the reduction of other costs covers the difference.

More recently, Woolf et al., (2015) assessed the feasibility of comparing therapies for word finding for persons with aphasia with four groups: remote therapy from University site, remote therapy from clinical site, in-person therapy, and attention control group. Woolf enrolled 21 persons with post-stroke aphasia and reported that participants gave positive ratings for the method of remote delivery. Participants in all therapy groups improved when compared to the attention-control groups but those who received remote therapy from the clinical site were most improved.

A descriptive feasibility study conducted by Azad et al., evaluated the use of VC for dementia management in rural areas. The study involved 99 patients who used VC from the Tri-County Mental Health site in Cornwall to see 32 health care providers at the Ottawa Hospital between Nov. 2006 and Nov. 2010. The patients were identified by a geriatrician as being medically stable with noncomplex conditions and presenting with mild cognitive impairment or mild dementia. Only 50 of the 99 patients (51\%) provided complete information on these measures. Most (92\%) of the patients felt they were able to communicate all desired information to their provider as they could during an in-person visit. The Majority (90\%) of patients and providers state they would be willing to use VC again. Of the 32 providers involved, 30 gave feedback and felt many of their appointments would have been canceled had the patient had to travel to the clinic, and 96\% felt the VC appointment gave them needed advise related to clinical decision making. Without technical difficulties, which 7 out of the 30 providers experienced, the VC system allowed them to monitor their patients more closely and with greater efficiency.

\subsection{Lifestyle Modification}

Some success has been reported with VC for group and lifestyle intervention programs aimed at preventing diabetes [49], improving obesity [32], and healthy relationship habits [42]. The diabetes prevention study had an on-site program $(\mathrm{n}=13)$ and a VC program $(\mathrm{n}=14)$ that were both 16-weeks in which people with risk factors for diabetes were asked to keep track of fat and calorie intake and record minutes of physical activity. In both groups over $45 \%$ of participants achieved the $7 \%$ weight loss goal although there was no significant difference between the groups. The implications from this study are that increased participation from multiple sites in a diabetes prevention program is possible. Obesity interventions, such as virtual group visits using VC may assist in overcoming barriers including access to care and yield positive clinical outcomes, such as a decrease in body mass index. Results from a VC study of relationships in women with HIV showed that participants $(n=4)$ liked the increased and equitable attention among group members as well as reportedly high levels of unity and togetherness in the group. All the women stated that they would prefer VC for the Healthy Relationship program as opposed to an in-person program.

\subsection{Oncology}

Oncology patients have also experienced benefits with use of VC. Tamara and colleagues (2015) conducted a 
pilot study of a mobile health Pain Coping Skills Training (PCST) protocol for 25 patients with persistent pain from breast, lung, colorectal or prostate cancer who live an average of 69 miles from the medical center. Participants received a tablet computer to access four pre-planned video-conferencing sessions. Thirty to 45 minute video conferencing sessions using skype from the therapists' office taught patients theoretical content on pain management and coached them through skills-based training including relaxation techniques. Of the $84 \%(\mathrm{~N}=$ 19) of patients who completed the study, all found it feasible and were satisfied with the program quality; $95 \%$ said it increased their understanding and over $90 \%$ reported pain management skill development. Significant reductions in pain severity, physical symptoms, psychological distress, and pain catastrophizing were found post-intervention.

Kitamura, Zurawel-Balaura \& Wong (2010) conducted a systematic review of the literature to evaluate the feasibility of assessing, monitoring, and managing oncology patients via VC. They identified 19 published articles of 15 clinical oncology patient groups: one small randomized controlled trial (RCT); 7 non-randomized with control groups, and 7 case studies, with a total of 709 VC study patients and 346 control patients. Inclusion criteria were that VC included two-way communication for monitoring and was used by physicians or nurses with cancer patients in real time with at least one reported clinical outcome. Reported outcomes included patient satisfaction (although no validated scales were included) and preference for VC consultation, costs, provider satisfaction and convenience. The study supported the feasibility of VC with effectiveness for assessing, monitoring, and managing care of oncology patients. Clinical outcomes were not compromised and time and cost were comparable or reduced.

\subsection{Follow-Up Care}

Video conferencing has also been used in older populations for follow-up care for common chronic conditions and acute exacerbations of chronic conditions. Woodend and colleagues (2008) conducted a RCT to evaluate the effect on healthcare resource use, morbidity, and quality of life, of a 3 month intervention that included VC enhanced telemonitoring for patients with heart failure (HF) or angina. The weekly VC intervention involved a nurse contact in addition to daily telephone transmission of weight, blood pressure and periodic electrocardiograms (EKG) and a 1-year end-of study assessment. Video conferencing in combination with home tele-monitoring was easy to use and had high satisfaction. There were no significant differences in physician visits between the VC and usual care groups. Primary outcome data documented 51\% reduced number of hospital readmissions and 61\% reduced days in hospital for patients with angina over 3 months and one year (reduced 45\%/ 21\%). Video conferencing was also an effective model of care for residents of long-term care facilities to enhance medical decision making for unscheduled conferencing with on call-physicians [23] and to facilitate consultation with specialty care [50].

\section{Discussion}

Although VC has been studied for the past 15 years, evidence of effectiveness is limited by a low number of RCTs with large numbers of participants. Small samples and methodological weaknesses of the studies are major limiters to generalizability of the findings. However, studies of VC have shown feasibility, acceptability, efficacy, and cost effectiveness. In addition, initial assessment of movement and mental health disorders using VC may be different from in person care. However, diagnosis and treatment ability have been found to be the same as in-person care. More research is needed with larger samples and randomization.

The outcomes of this systematic review are that studies are not adequately designed to address the question of whether "access" in rural areas is improved through VC because multiple factors could have influenced the results of the studies. In addition, technical difficulties from what may be outdated technology have limited evidence of mostly moderate quality. Perhaps qualitative research can be used to identify key factors needed to improve access and what components can be done using VC. This could provide a foundation for quantitative studies of VC looking at health care re-admission, co-morbidity, deaths, and cost-effectiveness.

Over the past 5 years, most users of VC have moved to secure web-based systems and away from slower ISDN connections. There is a gap in publication of VC studies in countries outside the United States. Twenty of the 27 studies are conducted in North America where access to faster internet connection and technology is more prevalent. Countries and rural areas that have significant disparity in access to technology and fast internet may find this shift problematic. 
Publication bias is a potential issue with this review. Much of the video conferencing technology is being developed by private industry and being used by clinical practices. Both of these groups may not have an academic purpose. Hence, much of the evidence of acceptability, feasibility and efficacy of video conferencing may not be published. This systematic review also contains clinical and methodological heterogeneity across studies. The types of participants, intervention and outcomes differ in each study and variability in study design occurs across studies. Statistical heterogeneity cannot be assessed in this article because a meta-analysis was not completed. Our objective was to complete a comprehensive review of the literature related to video conferencing so that we may conduct a practice change. The underlying systematic review of this literature does not yet lend itself to a meta-analysis. There are very few randomized controlled trials (RCTs) and the measures are quite different between the limited RCTs. However, we did not want to exclude articles from our review that were not RCTs as we believe they contribute meaningful information to our objective. In addition, this review provides insight on how to proceed both clinically and in future research.

All 8 RCTs used the comparators of in-person, usual care. Standard in-person usual care could vary from location to location and from country to country. No studies were found that compared different types of VC to determine the most effective method/type/intervention. The studies reviewed focused on patients from multiple populations with a differing chronic illnesses. While VC has been used in a wide range of health problems, reported studies using VC focus on one specific disease. VC has potential for use in patients with MCC and health disparities in multiple healthcare settings. Addressing the confounding co-morbid conditions experienced by many individuals living with chronic illness should be a priority. Future, prospective randomized controlled trials with adequate power that incorporate VC into Primary care and focus on MCC are warranted. Designing these trials will be complex and should be done using an organizing framework for developing complex interventions.

\section{Acknowledgements}

This project was supported by the Robert Wood Johnson Foundation Nurse Faculty Scholars Program. The Robert Wood Johnson Foundation Nurse Faculty Scholars Program is working to develop the next generation of national leaders in academic nursing through career development awards for outstanding junior nursing faculty. The program aims to strengthen the academic productivity and overall excellence of nursing schools by providing mentorship, leadership training and salary and research support to faculty.

\section{References}

[1] Bury, M. (1991) The Sociology of Chronic Illness: A Review of Research and Prospects. Sociology of Health and Illness, 13, 451-468. http://dx.doi.org/10.1111/j.1467-9566.1991.tb00522.x

[2] Mallow, J.A., Theeke, L.A., Barnes, E.R., Whetsel, T. and Mallow, B.K. (2014) Free Care Is Not Enough: Barriers to Attending Free Clinic Visits in a Sample of Uninsured Individuals with Diabetes. Open Journal of Nursing, 4, 912.

[3] Singh, G.K. and Siahpush, M. (2014) Widening Rural-Urban Disparities in All-Cause Mortality and Mortality from Major Causes of Death in the USA, 1969-2009. Journal of Urban Health, 91, 272-292.

[4] Bauer, U.E., Briss, P.A., Goodman, R.A. and Bowman, B.A. (2014) Prevention of Chronic Disease in the 21st Century: Elimination of the Leading Preventable Causes of Premature Death and Disability in the USA. The Lancet, 384, 45-52. http://dx.doi.org/10.1016/S0140-6736(14)60648-6

[5] Ward, B.W., Schiller, J.S. and Goodman, R.A. (2014) Peer Reviewed: Multiple Chronic Conditions Among US Adults: A 2012 Update. Preventing Chronic Disease, 2014, 11.

[6] Grembowski, D., Schaefer, J., Johnson, K.E., Fischer, H., Moore, S.L., Tai-Seale, M., et al. (2014) A Conceptual Model of the Role of Complexity in the Care of Patients with Multiple Chronic Conditions. Medical Care, 52, S7-S14.

[7] LeRoy, L., Bayliss, E., Domino, M., Miller, B.F., Rust, G., Gerteis, J., et al. (2014) The Agency for Healthcare Research and Quality Multiple Chronic Conditions Research Network: Overview of Research Contributions and Future Priorities. Medical Care, 52, S15-S22.

[8] Magnan, E.M., Palta, M., Mahoney, J.E., Pandhi, N., Bolt, D.M., Fink, J., et al. (2015) The Relationship of Individual Comorbid Chronic Conditions to Diabetes Care Quality. BMJ Open Diabetes Research \& Care, 3, e000080.

[9] Tinetti, M.E. and Basu, J. (2014) Research on Multiple Chronic Conditions: Where We Are and Where We Need to Go. Medical Care, 52, S3-S6.

[10] Utz, S.W. (2008) Diabetes Care among Rural Americans. Annual Review of Nursing Research, 26, 3-39. 
[11] Mallow, J.A., Theeke, L.A., Barnes, E.R. and Whetsel, T. (2015) Examining Dose of Diabetes Group Medical Visits and Characteristics of the Uninsured. Western Journal of Nursing Research, 37, 1033-1061.

[12] Shi, L., Chen, C.-C., Nie, X., Zhu, J. and Hu, R. (2014) Racial and Socioeconomic Disparities in Access to Primary Care among People with Chronic Conditions. The Journal of the American Board of Family Medicine, 27, 189-198.

[13] Khairat, S., Wijesinghe, N., Wolfson, J., Scott, R. and Simkus, R. (2014) Building a Multicenter Telehealth Network to Advance Chronic Disease Management. Integrating Information Technology and Management for Quality of Care, 202, 299.

[14] Lukacs, M.E. (1998) Infinitely Expandable Real-Time Video Conferencing System. Google Patents.

[15] Liberati, A., Altman, D.G., Tetzlaff, J., Mulrow, C., Gøtzsche, P.C., Ioannidis, J.P., et al. (2009) The PRISMA Statement for Reporting Systematic Reviews and Meta-Analyses of Studies That Evaluate Health Care Interventions: Explanation and Elaboration. Annals of Internal Medicine, 151, W-65-W-94. http://dx.doi.org/10.7326/0003-4819-151-4-200908180-00136

[16] Rosswurm, M.A. and Larrabee, J.H. (1999) A Model for Change to Evidence-Based Practice. Image: The Journal of Nursing Scholarship, 31, 317-322. http://dx.doi.org/10.1111/j.1547-5069.1999.tb00510.x

[17] Greenhalgh, T., Robert, G., Macfarlane, F., Bate, P. and Kyriakidou, O. (2004) Diffusion of Innovations in Service Organizations: Systematic Review and Recommendations. Milbank Quarterly, 82, 581-629. http://dx.doi.org/10.1111/j.0887-378X.2004.00325.x

[18] Loh, P. (2008) Telemedicine and Alzheimer's Disease from Studio-Based Videoconferencing to Mobile Handheld Cell Phones. Journal of Brain Disease, 1, 39-43.

[19] Chatrath, V., Attri, J.P. and Chatrath, R. (2010) Telemedicine and Anaesthesia. Indian Journal of Anaesthesia, 54, 199204. http://dx.doi.org/10.4103/0019-5049.65357

[20] Alanee, S., Dynda, D., Levault, K., Mueller, G., Sadowski, D., Wilber, A., et al. (2014) Delivering Kidney Cancer Care in Rural Central and Southern Illinois: A Telemedicine Approach. European Journal of Cancer Care, 23, 739744. http://dx.doi.org/10.1111/ecc.12248

[21] Foucher, Y., Meurette, A., Daguin, P., Bonnaud-Antignac, A., Hardouin, J.-B., Chailan, S., et al. (2015) A Personalized Follow-Up of Kidney Transplant Recipients Using Video Conferencing Based on a 1-Year Scoring System Predictive of Long Term Graft Failure (TELEGRAFT Study): Protocol for a Randomized Controlled Trial. BMC Nephrology, 16, 6. http://dx.doi.org/10.1186/1471-2369-16-6

[22] Strachan, M., Gros, D.F., Yuen, E., Ruggiero, K.J., Foa, E.B. and Acierno, R. (2012) Home-Based Telehealth to Deliver Evidence-Based Psychotherapy in Veterans with PTSD. Contemporary Clinical Trials, 33, 402-409. http://dx.doi.org/10.1016/j.cct.2011.11.007

[23] Weiner, M., Schadow, G., Lindbergh, D., Warvel, J., Abernathy, G., Perkins, S.M., et al. Editors. (2002) Conducting a Study of Internet-Based Video Conferencing for Assessing Acute Medical Problems in a Nursing Facility. American Medical Informatics Association, Proceedings of the AMIA Symposium, 874-878.

[24] Achey, M., Aldred, J.L., Aljehani, N., Bloem, B.R., Biglan, K.M., Chan, P., et al. (2014) The Past, Present, and Future of Telemedicine for Parkinson's Disease. Movement Disorders, 29, 871-883. http://dx.doi.org/10.1002/mds.25903

[25] Wong, L., Martin-Khan, M., Rowland, J., Varghese, P. and Gray, L. (2011) Reliability of the Rowland Universal Dementia Assessment Scale (RUDAS) via Video Conferencing. International Journal of Geriatric Psychiatry, 26, 988989. http://dx.doi.org/10.1002/gps.2658

[26] Catic, A.G., Mattison, M.L., Bakaev, I., Morgan, M., Monti, S.M. and Lipsitz, L. (2014) ECHO-AGE: An Innovative Model of Geriatric Care for Long-Term Care Residents with Dementia and Behavioral Issues. Journal of the American Medical Directors Association, 15, 938-942. http://dx.doi.org/10.1016/j.jamda.2014.08.014

[27] Nápoles, A.M., Santoyo-Olsson, J., Karliner, L.S., O’Brien, H., Gregorich, S.E. and Pérez-Stable, E.J. (2010) Clinician Ratings of Interpreter Mediated Visits in Underserved Primary Care Settings with Ad hoc, In-Person Professional, and Video Conferencing Modes. Journal of Health Care for the Poor and Underserved, 21, 301-317. http://dx.doi.org/10.1353/hpu.0.0269

[28] Rowe, M., Rosenheck, R., Stern, E. and Bellamy, C. (2014) Video Conferencing Technology in Research on Schizophrenia: A Qualitative Study of Site Research Staff. Psychiatry, 77, 98-102. http://dx.doi.org/10.1521/psyc.2014.77.1.98

[29] Schutte, J., Gales, S., Filippone, A., Saptono, A., Parmanto, B. and McCue, M. (2012) Evaluation of a Telerehabilitation System for Community-Based Rehabilitation. International Journal of Telerehabilitation, 4, 15-24. http://dx.doi.org/10.5195/ijt.2012.6092

[30] Amarendran, V., George, A., Gersappe, V., Krishnaswamy, S. and Warren, C. (2011) The Reliability of Telepsychiatry for a Neuropsychiatric Assessment. Telemedicine and E-Health, 17, 223-225. http://dx.doi.org/10.1089/tmj.2010.0144 
[31] Azad, N., Amos, S., Milne, K. and Power, B. (2012) Telemedicine in a Rural Memory Disorder Clinic-Remote Management of Patients with Dementia. Canadian Geriatrics Journal, 15, 96-100. http://dx.doi.org/10.5770/cgj.15.28

[32] Azar, K.M., Aurora, M., Wang, E.J., Muzaffar, A., Pressman, A. and Palaniappan, L.P. (2015) Virtual Small Groups for Weight Management: An Innovative Delivery Mechanism for Evidence-Based Lifestyle Interventions among Obese Men. Translational Behavioral Medicine, 5, 37-44. http://dx.doi.org/10.1007/s13142-014-0296-6

[33] Chua, R., Craig, J., Wootton, R. and Patterson, V. (2001) Randomised Controlled Trial of Telemedicine for New Neurological Outpatient Referrals. Journal of Neurology, Neurosurgery \& Psychiatry, 71, 63-66. http://dx.doi.org/10.1136/jnnp.71.1.63

[34] Dorsey, E.R., Venkataraman, V., Grana, M.J., Bull, M.T., George, B.P., Boyd, C.M., et al. (2013) Randomized Controlled Clinical Trial of "Virtual House Calls” for Parkinson Disease. JAMA Neurology, 70, 565-570. http://dx.doi.org/10.1001/jamaneurol.2013.123

[35] Grady, B.J. (2002) A Comparative Cost Analysis of an Integrated Military Telemental Health-Care Service. Telemedicine Journal and E-health, 8, 293-300. http://dx.doi.org/10.1089/15305620260353180

[36] Grady, B.J. and Melcer, T. (2005) A Retrospective Evaluation of Telemental Healthcare Services for Remote Military Populations. Telemedicine Journal \& E-Health, 11, 551-558. http://dx.doi.org/10.1089/tmj.2005.11.551

[37] Hailey, D., Roine, R. and Ohinmaa, A. (2008) The Effectiveness of Telemental Health Applications: A Review. Canadian Journal of Psychiatry Revue Canadienne de Psychiatrie, 53, 769-778.

[38] Khatri, N., Marziali, E., Tchernikov, I. and Shepherd, N. (2014) Comparing Telehealth-Based and Clinic-Based Group Cognitive Behavioral Therapy for Adults with Depression and Anxiety: A Pilot Study. Clinical Interventions in Aging, 9, 765-770. http://dx.doi.org/10.2147/CIA.S57832

[39] Kitamura, C., Zurawel-Balaura, L. and Wong, R. (2010) How Effective Is Video Consultation in Clinical Oncology? A Systematic Review. Current Oncology, 17, 17-27.

[40] Lipman, E.L., Kenny, M. and Marziali, E. (2011) Providing Web-Based Mental Health Services to At-Risk Women. BMC Women's Health, 11, 38-47. http://dx.doi.org/10.1186/1472-6874-11-38

[41] Man, D., Soong, W., Tam, S. and Hui-Chan, C. (2005) A Randomized Clinical Trial Study on the Effectiveness of a Tele-Analogy-Based Problem-Solving Programme for People with Acquired Brain Injury (ABI). NeuroRehabilitation, 21, 205-217.

[42] Marhefka, S.L., Iziduh, S., Fuhrmann, H.J., Lopez, B., Glueckauf, R., Lynn, V., et al. (2013) Internet-Based Video-Group Delivery of Healthy Relationships-A "Prevention with Positives" Intervention: Report on a Single Group Pilot Test among Women Living with HIV. AIDS Care, 25, 904-909. http://dx.doi.org/10.1080/09540121.2013.793266

[43] Martin-Khan, M., Flicker, L., Wootton, R., Loh, P.-K., Edwards, H., Varghese, P., et al. (2012) The Diagnostic Accuracy of Telegeriatrics for the Diagnosis of Dementia via Video Conferencing. Journal of the American Medical Directors Association, 13, 487.e19-487.e24. http://dx.doi.org/10.1016/j.jamda.2012.03.004

[44] Marziali, E. and Donahue, P. (2006) Caring for Others: Internet Video-Conferencing Group Intervention for Family Caregivers of Older Adults with Neurodegenerative Disease. The Gerontologist, 46, 398-403. http://dx.doi.org/10.1093/geront/46.3.398

[45] Marziali, E. and Garcia, L.J. (2011) Dementia Caregivers’ Responses to 2 Internet-Based Intervention Programs. American Journal of Alzheimer's Disease and Other Dementias, 26, 36-43. http://dx.doi.org/10.1177/1533317510387586

[46] Norman, S. (2006) The Use of Telemedicine in Psychiatry. Journal of Psychiatric and Mental Health Nursing, 13, 771-777. http://dx.doi.org/10.1111/j.1365-2850.2006.01033.x

[47] Somers, T.J., Abernethy, A.P., Edmond, S.N., Kelleher, S.A., Wren, A.A., Samsa, G.P., et al. (2015) A Pilot Study of a Mobile Health Pain Coping Skills Training Protocol for Patients with Persistent Cancer Pain. Journal of Pain and Symptom Management, 50, 553-558. http://dx.doi.org/10.1016/j.jpainsymman.2015.04.013

[48] Temple, V., Drummond, C., Valiquette, S. and Jozsvai, E. (2010) A Comparison of Intellectual Assessments over Video Conferencing and In-Person for Individuals with ID: Preliminary Data. Journal of Intellectual Disability Research, 54, 573-577. http://dx.doi.org/10.1111/j.1365-2788.2010.01282.x

[49] Vadheim, L.M., McPherson, C., Kassner, D.R., Vanderwood, K.K., Hall, T.O., Butcher, M.K., et al. (2010) Adapted Diabetes Prevention Program Lifestyle Intervention Can Be Effectively Delivered through Telehealth. The Diabetes Educator, 36, 651-656. http://dx.doi.org/10.1177/0145721710372811

[50] Wakefield, B.J., Buresh, K.A., Flanagan, J.R. and Kienzle, M.G. (2004) Interactive Video Specialty Consultations in Long-Term Care. Journal of the American Geriatrics Society, 52, 789-793. http://dx.doi.org/10.1111/j.1532-5415.2004.52220.x

[51] Woodend, A.K., Sherrard, H., Fraser, M., Stuewe, L., Cheung, T. and Struthers, C. (2008) Telehome Monitoring in Patients with Cardiac Disease Who Are at High Risk of Readmission. Heart \& Lung: The Journal of Acute and Critical 
Care, 37, 36-45. http://dx.doi.org/10.1016/j.hrtlng.2007.04.004

[52] Woolf, C., Caute, A., Haigh, Z., Galliers, J., Wilson, S., Kessie, A., et al. (2016) A Comparison of Remote Therapy, Face to Face Therapy and an Attention Control Intervention for People with Aphasia: A Quasi-Randomised Controlled Feasibility Study. Clinical Rehabilitation, 30, 359-373. http://dx.doi.org/10.1177/0269215515582074

[53] Lewis, J., Boian, R. and Burdea, G. (2003) Real-Time Web-Based Telerehabilitation Monitoring. Medicine Meets Virtual Reality 11, NextMed: Health Horizon, January 2003, 190-192.

[54] Peel, N.M., Russell, T.G. and Gray, L.C. (2011) Feasibility of Using an In-Home Video Conferencing System in Geriatric Rehabilitation. Journal of Rehabilitation Medicine, 43, 364-366. http://dx.doi.org/10.2340/16501977-0675

\section{List of Abbreviations}

$\mathrm{ABI}=$ Acquired Brain Injury

AIMS = Abnormal Involuntary Movement Scale

BDI-II = Beck Depression Inventory Version 2

BMI $=$ Body Mass Index

$\mathrm{BP}=$ Blood Pressure

$\mathrm{CBT}=$ Cognitive behavioral Therapy

CES-D = Center for Epidemiologic Studies Depression Scale

CFT $=$ Clock Face Test

$\mathrm{DAD}=$ Disability Assessment for Dementia

$\mathrm{EKG}=$ Electrocardiograms

FS $=$ Finger Stick

$\mathrm{HF}=$ Hear Failure

HIV $=$ Human Immunodeficiency Virus

ID = Intellectual Disability

$\mathrm{IQ}=$ Intelligence Quotient

ISDN = Integrated Services Digital Network

IQCODE = Informant Questionnaire on Cognitive Decline in the Elderly

GSD-15 = Geriatric Depression Scale-15 Questions

MCC $=$ Multiple Chronic Conditions

MMSE $=$ Mini-Mental State Examination

NPI-Q $=$ Neuropsychiatric Inventory Short form

OCD = Obsessive Compulsive Disorder

$\mathrm{PC}=$ Primary Care provider

PCST $=$ Pain Coping Skills Training

PTSD $=$ Post Traumatic Stress Disorder

RCT $=$ Randomized controlled Trial

RUDAS = Rowland Universal Dementia Assessment Scale

SF36 $=$ Short Form Health Survey 36 questions

$\mathrm{VA}=$ Veterans Administration

$\mathrm{VC}=$ Video Conferencing

VMI = Beery-Buktenice Developmental Test of Visual-Motor Integration-IV

WASI $=$ Wechsler Abbreviated Scale of Intelligence 